Representaciones sociales de estudiantes universitarios en Santiago de Chile sobre el consumo de marihuana Ignacio Abarca Saavedra, Sarah Baïz

Revista Argentina de Estudios de Juventud, (14), e038, 2020

ISSN 1852-4907 | https://doi.org/10.24215/18524907e038

https://perio.unlp.edu.ar/ojs/index.php/revistadejuventud

FPyCS | Universidad Nacional de La Plata

La Plata | Buenos Aires | Argentina

\title{
REPRESENTACIONES SOCIALES DE ESTUDIANTES UNIVERSITARIOS EN SANTIAGO DE CHILE SOBRE EL CONSUMO DE MARIHUANA
}

\author{
Social Representations of University Students \\ in Santiago De Chile on the Consumption of Marijuana
}

Ignacio Abarca Saavedra

igabarcs@gmail.com

https://orcid.org/0000-0003-2470-3926

\section{Sarah Baïz}

ziaboh@gmail.com

https://orcid.org/0000-0001-6509-1626

Departamento de Sociología

Universidad Alberto Hurtado | Chile

\section{Palabras clave}

representación social estudiantes universitarios consumo de marihuana identidad

\section{Keywords}

social representation university students marijuana use identity

\section{Resumen}

Esta investigación analiza las representaciones sociales sobre el consumo de drogas de estudiantes universitarios en Santiago de Chile. El fenómeno se aborda desde un enfoque cualitativo mediante la realización de entrevistas semiestructuradas. Los resultados muestran que las representaciones sociales construyen un tejido donde convergen estigmas y estatus hacia consumidores. Se devela que la representación social sobre la marihuana se vincula a la amistad, el relajo y la diversión, y que a través de su consumo se impregnan y se resinifican territorios que a su vez se utilizan como espacios de resistencia.

\section{Abstract}

This research analyzed the social representations of drug use by university students in Santiago, Chile. The phenomenon was approached from a qualitative approach, conducting semi-structured interviews. The results showed that social representations build a fabric where stigmas and status converge towards consumers. It is revealed that the social representation of marijuana is linked to friendship, relaxation and fun, and that through its consumption, territories that in turn are used as resistance spaces are impregnated and reshaped. 


\title{
REPRESENTACIONES SOCIALES \\ DE ESTUDIANTES UNIVERSITARIOS \\ EN SANTIAGO DE CHILE \\ SOBRE EL CONSUMO DE MARIHUANA
}

\author{
Por Ignacio Abarca Saavedra y Sarah Baïz
}

De acuerdo con la Organización de Estados Americanos (OEA) y la Comisión Interamericana para el Control del Abuso de Drogas (CICAD) (2019), el uso de marihuana y de tabaco por parte de jóvenes chilenos sitúa al país en el primer lugar de consumo juvenil de estas drogas en relación con otros países de América y el Caribe. En este contexto, es importante contemplar que la juventud es la etapa más vulnerable en relación con el consumo de drogas (Faúndez \& Vinet, 2009) y que corresponde al grupo etario en el que más se concentra el uso de drogas (Ramírez, Cunningham, Brands, Strike \& Miotto, 2009).

La complejidad del fenómeno sobre el consumo de drogas impregna la esfera social con diferentes producciones simbólicas que se manifiestan en los discursos y en las acciones en relación con el uso de estupefacientes. Además, la producción simbólica no necesariamente tiene significados homogéneos; estos pueden variar según la posición de las personas en el entramado social (lbáñez, 1988).

El consumo juvenil de drogas en Chile ha sido abordado desde diversas perspectivas. Sin embargo, escasas investigaciones se enfocan en las representaciones sociales sobre el consumo de drogas. Conocer las representaciones sociales de jóvenes respecto al consumo es necesario, ya que estas influencian la construcción de su identidad, las formas en que se relacionan con las drogas -en particular, con la marihuana-, cómo hacen uso de los espacios y las justificaciones de sus acciones a partir de sus percepciones, entendidas como sentido común (Araya, 2002). 
En este estudio se analizan las representaciones sociales sobre el consumo situado en un espacio específico. Este lugar es conocido por los jóvenes como Erasmo Escala, y se ubica en la calle del mismo nombre, entre las calles Almirante Barroso y Cienfuegos, en la comuna de Santiago (Región Metropolitana), a un costado de la Universidad Alberto Hurtado (UAH). Se tomó como referencia esta zona debido a que todo el día e, incluso, en horas de la noche existe flujo de personas, y es habitual el consumo de alimentos, marihuana, tabaco y alcohol, acompañado de juegos de cartas, malabares, música y baile.

El artículo comienza con una revisión de antecedentes que muestra cómo se ha estudiado el fenómeno del consumo juvenil de drogas. En esta sección se abordan los diferentes enfoques y corrientes científicas que han estudiado el uso de drogas y se exponen las principales conclusiones obtenidas. También se profundizan cifras sobre el consumo juvenil chileno y se explican las formas en que se ha estudiado el fenómeno en el país. Finalmente, se expone de manera breve una perspectiva de estudio específica, el estudio de las representaciones sociales acerca del consumo de drogas.

En el segundo apartado se desarrollan las categorías conceptuales para abordar el fenómeno. Se introducen nociones sobre la construcción de la realidad social y se definen los alcances del concepto de representación social, al tiempo que se detalla, y se conceptualiza, su influencia en la construcción de la identidad. A continuación, se explica la forma en que es abordado el fenómeno en estudio: desde un enfoque cualitativo que privilegia el uso de entrevistas semiestructuradas y que aplica el análisis de contenido cualitativo para su procesamiento. En el apartado siguiente se presentan los resultados $\mathrm{y}$, finalmente, se exponen las conclusiones del estudio.

\section{Antecedentes}

El uso de drogas es más común entre los 18 y los 29 años que en otra franja etaria, es decir durante la adultez emergente (Naciones Unidas, 2013, en Cazenave, Saavedra, Huerta, Mendoza \& Aguirre, 2017). Dicha adultez emergente coincide con la etapa universitaria (Barrera-Herrera \& Vinet, 2017), período en el cual los/as jóvenes son cada vez más vulnerables a consumir drogas (López Pinedo, 2012). 
La prevalencia del consumo juvenil puede entenderse desde tres enfoques: 1) es una nueva etapa en la que se tiende a buscar nuevas experiencias y una nueva identidad (Ovejero, 2000; Silva, 2002); 2) existe un contexto de mayor libertad, ya que el/la joven comienza a desenvolverse en espacios en los que prevalece la autonomía (CONACE, 2008; Henao, 2011); 3) existen factores psicosociales, relaciones interpersonales y actividades que los/as jóvenes realizan que influyen en su consumo (Cáceres, Salazar, Varela \& Tovar, 2006; Jiménez-Muro Franco, Beamonte, Marqueta, Gargallo \& Nerin, 2009; López Pinedo, 2012).

El primer enfoque tematiza la búsqueda de experiencias y de identidad como un predictor del consumo de drogas. Anastasio Ovejero Bernal (2000) afirma que la búsqueda de una identidad personal y social impulsa a los/as jóvenes a integrarse a diferentes grupos, en los cuales existen señas identitarias como sucede con el consumo de droga. Esto es importante debido a que la identidad influye en cómo interpretamos el entorno y a nosotros mismos, a la vez que se obtiene a través del reconocimiento de otros (Ovejero Bernal, 2000). La relación entre identidad y consumo de drogas se intensifica en la universidad, ya que en este espacio se potencia el vínculo entre alcohol y diversión, o alcohol y evasión del estrés (López Pinedo, 2012).

En relación con el segundo enfoque, referido a la libertad y la autonomía en el contexto universitario, Silvia Henao (2011) y el Consejo Nacional para el Control de Estupefacientes (CONACE, 2008) muestran que los/as estudiantes asimilan el espacio universitario como un lugar de libertad, autonomía, diversión y tolerancia. En este escenario, el consumo de drogas se entiende como un elemento más del entramado de relaciones y, por lo tanto, es naturalizado. Asimismo, el uso de drogas es entendido como un elemento facilitador de las relaciones, como un distractor y un identificador de grupos en un contexto donde priman aspectos académicos (Henao, 2011).

El tercer enfoque identifica factores asociados al consumo juvenil de drogas. Algunos atienden a elementos psicosociales, como la autoestima o el autocontrol (Cáceres y otros, 2006); otros, los contextos de relaciones interpersonales, como el consumo de pares o de familiares (Jiménez-Muro Franco y otros, 2009). Finalmente, también se ha tematizado el factor ingreso, el cual se incrementa en esta etapa y aumenta la posibilidad de adquirir sustancias (Jiménez-Muro Franco y otros, 2009). 
Según la OEA y la CICAD (2019), el consumo juvenil de drogas en Chile posiciona al país en los primeros lugares en relación con el resto del continente, especialmente respecto del consumo de marihuana y de tabaco, drogas que alcanzan el primer lugar respecto al consumo juvenil en América y el Caribe.

\begin{tabular}{|l|c|c|c|c|c|c|}
\hline & \multicolumn{2}{|c|}{ Marihuana (en el último año) } & \multicolumn{3}{c|}{ Tabaco (en el último mes) } \\
\hline Rango etario & \multicolumn{3}{|c|}{ Entre 18 y 34 años } & \multicolumn{3}{c|}{ Entre 18 y 34 años } \\
\hline Países & Chile & $\begin{array}{c}\text { Estados } \\
\text { Unidos }\end{array}$ & Canadá & Chile & Uruguay & Argentina \\
\hline $\begin{array}{l}\text { Porcentaje } \\
\text { de consumo }\end{array}$ & $\begin{array}{c}\text { Entre } 25 \\
\text { y 30\% }\end{array}$ & $\begin{array}{c}\text { Entre } 25 \\
\text { y 30\% }\end{array}$ & $25 \%$ & $\begin{array}{c}\text { Entre } 35 \\
\text { y } 40 \%\end{array}$ & $\begin{array}{c}\text { Entre } 30 \\
\text { y } 35 \%\end{array}$ & $\begin{array}{c}\text { Entre } 30 \\
\text { y } 35 \%\end{array}$ \\
\hline
\end{tabular}

Tabla 1 | Consumo porcentual de marihuana y tabaco en jóvenes por países Fuente: elaboración propia a partir de OEA \& CICAD (2019)

En este contexto, el Servicio Nacional para la Prevención y Rehabilitación del Consumo de Drogas y Alcohol (SENDA, 2019a) afirma que 33\% de los/as jóvenes universitarios chilenos ha consumido marihuana durante el último mes, dentro de los cuales 36,1\% declara haber consumido más de diez días en ese período. Respecto al tabaco, 16,5\% de los/as jóvenes de entre 19 a 25 años de edad declara consumirlo diariamente (SENDA, 2017).

De acuerdo con las cifras publicadas por SENDA (2017, 2019a), la marihuana es considerada una droga de inicio en el consumo juvenil. Esta categorización se justifica debido a que su consumo es transversal a todos los grupos de identidad, nivel socioeconómico, edad o tipos de consumo cultural (CONACE, 2008).

Asimismo, se categorizan diferentes tipologías de jóvenes y de universitarios/as en relación con el uso de drogas, lo que demuestra que existen diferentes formas de relacionarse con las drogas según el tipo de personalidad del/de la joven (Faúndez \& Vinet, 2009), el tipo de carrera que estudie e, incluso, el año universitario que curse (Morales, Del Valle, Belmar, Orellana, Soto \& Ivanovic, 2011).

Otro enfoque importante corresponde a investigaciones que muestran la relación entre normas percibidas por los/as estudiantes sobre el consumo de pares y el consumo personal (Cazenave y otros, 2017; Ramírez y otros, 2009). Estos estudios 
demuestran una relación directa entre la percepción que se tiene de las reglas de consumo de compañeros/as (cuánto creen que consumen) y el consumo propio, lo que se traduce en un factor de riesgo.

Finalmente, otras investigaciones muestran la relación entre consumo juvenil de drogas y violencia, y concluyen que existe una relación entre el consumo de drogas y la tendencia a enfrascarse en riñas callejeras, en el caso de los varones, y a cometer suicidio, en el caso de las mujeres (Rodríguez, Fernández, Hernández \& Ramírez, 2006). Además, aumentan las posibilidades de agredir y/o de ser agredido en una relación de noviazgo (Saldivia \& Vizcarra, 2012; Vázquez \& Castro, 2009).

Los estudios sobre el consumo de droga en Chile dan cuenta de las diversas formas en que los/as jóvenes se relacionan con ellas, las cuales dependen, principalmente, del espacio que estos/as ocupen en el entramado de relaciones sociales. Conforme a esta idea, y como se desarrollará a continuación, existen investigaciones que exponen las formas de representación social que los/as jóvenes poseen sobre el consumo de drogas (Bulla, Vásquez, Güichá, Manrique-Abril \& Ospina, 2010; Camarotti \& Kornblit, 2005; CONACE, 2008; García, Torres, Barrios, Vanessa, Rubio y Ugarte, 2010; Henao, 2011).

\section{Entramado de relaciones y consumo:}

estudios sobre la representación social

Durante los años noventa, en Chile y en el resto del continente, las investigaciones sobre juventud analizaron la producción de políticas públicas enfocadas en jóvenes. Estas políticas se centraron en cuatro ejes: violencia, prevención de enfermedades de trasmisión sexual, apoyo en la socialización de grupos de pares y problemas relacionados con la socialización (Aguilera Ruiz, 2009). En este contexto, uno de los escasos estudios publicados en Chile que delimita las representaciones sociales que poseen los/as jóvenes acerca del consumo de droga fue realizado por el CONACE (2008) y se titula «Representaciones sociales sobre el consumo de drogas de la(s) juventud(es) chilena».

Este trabajo demostró un uso diferencial de espacios y de drogas según el grupo de pertenencia de los/as jóvenes; así, algunos grupos utilizan espacios como las aceras, las discotecas, los bares o las casas particulares, al tiempo que eligen una 
droga u otra, o un tipo diferente de la misma (por ejemplo, marihuana prensada o natural, o el tipo de alcohol ingerido). La investigación expone, además, diferentes valoraciones sobre las drogas según su función: diversión, estatus, extender la noche o fraternización.

Otros países latinoamericanos también han estudiado las representaciones sociales sobre el consumo de drogas. Un ejemplo es el trabajo «Representaciones sociales del consumo de drogas en un contexto universitario, Medellín, Colombia, 2000» (Henao, 2011), donde se identifican las representaciones sociales que poseen estudiantes y trabajadores/as de una universidad de Colombia. La investigación concluye que el uso de drogas es un elemento socializador y facilitador de las relaciones sociales e, incluso, que permite la aceptación de los/as jóvenes al interior de grupos estudiantiles (Henao, 2011).

Un segundo ejemplo corresponde al estudio argentino «Representaciones sociales y prácticas del consumo de éxtasis» (Camarotti \& Kornblit, 2005). En este trabajo se analizan las representaciones sociales de jóvenes que participan en raves de Buenos Aires acerca del consumo de éxtasis. Los resultados vinculan esta droga a su funcionalidad: existe un nexo entre estatus y consumo, al tiempo que es entendida como una sustancia útil para soportar los requerimientos de estas fiestas (bailar, incluso, hasta el día siguiente). Debido a su utilidad y a lo esporádico de estos eventos no se perciben peligros al consumirla (Camarotti \& Kornblit, 2005).

Finalmente, en Colombia también se han analizado las representaciones sociales de jóvenes sobre el consumo de marihuana. Estas investigaciones reconocen una doble valoración del consumo: en situaciones estresantes y en situaciones de distensión (García y otros, 2010). Además, afirman que los/as jóvenes distinguen en el discurso social un hostigamiento, debido a una visión negativa de la marihuana y de quienes la consumen. Asimismo, relacionan su uso con sensaciones placenteras y terapéuticas (Bulla y otros, 2010).

Las investigaciones presentadas demuestran los diversos enfoques desde los cuales se ha abordado, tanto a nivel nacional como internacional, el consumo juvenil de drogas, ya sea en espacios académicos o en grupos de pertenencia.

A pesar de esta atención, es llamativa la baja producción académica focalizada en las representaciones sociales juveniles sobre el consumo de drogas en Chile, siendo uno de los escasos intentos el mencionado estudio del CONACE (2008), del cual han 
pasado once años de su publicación. La incertidumbre ante esta falta de investigaciones aumenta debido al alto consumo de los/as jóvenes chilenos/as, quienes lideran el uso de marihuana y de tabaco en América y el Caribe (OEA \& CICAD, 2019).

De acuerdo a Henao (2011), en su mayoría, los/as jóvenes actúan sobre la base del sentido común (en oposición al conocimiento científico), incluso en contextos universitarios, por lo que la relación con la marihuana se genera a partir de creencias y de prácticas desde lo cotidiano. En consecuencia, las representaciones sociales generadas desde el sentido común de los/as jóvenes habitualmente desbordan los discursos y la investigación institucional que busca mostrar los peligros del consumo y, con ello, controlarlo eficazmente.

El consumo de drogas es parte de la vida social, especialmente en la juventud, por lo que se valida en el ámbito universitario (Cazenave y otros, 2017; Henao, 2011; Ramírez y otros, 2009). Comprender cómo los/as jóvenes representan el consumo es fundamental para desarrollar con ellos/as relaciones prósperas desde las instituciones, ya que estas representaciones delimitan cómo van a actuar en relación con la marihuana. Finalmente, conocer las representaciones sociales puede brindar orientaciones para la aplicación de una política eficaz de autocuidado y/o de prevención.

\section{La realidad: expresión objetiva de la intersubjetividad}

Para comprender el concepto de representación social, es pertinente revisar cómo diversos autores definen la realidad, ya que las representaciones sociales se enmarcan desde, o al menos comparten, estas nociones. Al respecto, Peter Berger y Thomas Luckmann (1968) explican que la realidad se construye socialmente mediante la interacción y la comunicación cotidiana de las personas. Los autores entienden que la habituación de conductas en el tiempo y su externalización mediante el lenguaje han permitido una objetivación y una significación de estos procesos. Tales significaciones facilitan su institucionalización, mediante la creación de justificaciones que permiten que permanezcan en el tiempo. 
Conforme a estas nociones, Berger y Luckmann (1968) explican que el lenguaje permite acumular conocimientos, gracias a lo cual se producen objetivaciones, que si bien en algún momento se generaron «cara a cara» con el tiempo este vínculo se desvanece debido a la ampliación del alcance social de las objetivaciones, que se vuelven anónimas. Sobre estas objetivaciones se construyen instituciones sociales que orientan las conductas de las personas. De este modo, se comparte una intersubjetividad entre los sujetos que hayan integrado estas objetivaciones a sus mundos cotidianos, formando parte del sentido común.

Considerando estas objetivaciones, Berger y Luckmann (1968) explican el motivo por el cual las personas asimilan la cotidianeidad ordenada, ya que la realidad presenta objetividad (entregada socialmente y de manera intersubjetiva) antes de que estos individuos aparezcan en escena. Esta objetividad se aprehende mediante la socialización, lo que no supone que las personas no apoyen en su construcción, ya que pueden externalizar sensaciones, experiencias y sentidos. No obstante, toda producción se genera en y a partir de este marco intersubjetivo denominado realidad social.

Los conocimientos, en tanto, no se encuentran distribuidos de manera homogénea. El hecho de que nos integremos a una categoría social determina la elaboración que realizamos de nuestra realidad. Así, la especificidad de los conocimientos acerca de objetivaciones y su aprehensión dependen, en parte, del lugar que la persona ocupe en el entramado social (Bourdieu, Passeron, Melendres \& Subirats, 1981; Ibáñez, 1988).

\section{Representación social y vida cotidiana}

El concepto de representación social proviene de Sergio Moscovici (1979) y fue posteriormente trabajado por autores como Denise Jodelet (1984), María Banchs (1986) y Jean-Claude Abric (2001). En las ciencias sociales, la conceptualización de las representaciones sociales se valoriza debido a que unifica lo individual y lo colectivo, lo simbólico y lo social, lo subjetivo y lo intersubjetivo (Araya, 2002).

El concepto de representación social es definido por Moscovici (1979) como una modalidad de conocimiento que permite la generación de comportamientos y la comunicación entre personas. Además, posibilita que los sujetos hagan inteligible 
la realidad física y social, y facilita que se integren a relaciones cotidianas de intercambios. En esta misma línea, Jodelet (1984) agrega que el campo de la representación social se orienta hacia los saberes que posee el sentido común o conocimiento ingenuo (en contraste con el conocimiento científico).

De igual manera, Banchs (1986) explica que las representaciones sociales se manifiestan a través de discursos espontáneos, que contienen valores, actitudes, creencias y opiniones enmarcadas en normas sociales. De allí que su estudio permita comprender los símbolos, los significados y las formas interpretativas que las personas utilizan en su relación con objetos.

Considerar las nociones de Banchs (1986) resulta de suma importancia debido a que se expone claramente que la interacción se genera en relación con objetos y que las representaciones sociales se encuentran delimitadas por un marco normativo. En este caso, el objeto será la marihuana y el marco normativo dependerá de los códigos o las significaciones que posean los/as jóvenes, las instituciones y los espacios en donde estos/as se desenvuelvan.

Se observa que el concepto de representación social comparte nociones acerca de la construcción de la realidad que realizan los propios actores. Entonces, la representación social es construida a través de la interacción entre sujetos en relación con objetos en un proceso comunicativo o de acciones (de las cuales se derivan emociones, sentimientos, entre otros) que pueden ser externalizadas. A su vez, esta representación construye (o norma) el comportamiento de las personas en relación con objetos en un espacio-tiempo determinado; las representaciones sociales contribuyen a configurar la realidad, pero también ayudan a construir el objeto del cual son una representación (Ibáñez, 1988, en Araya, 2002).

Respecto de las funciones de las representaciones sociales, Moscovici (1979) reconoce tres: hacer inteligible la realidad social, permitir la comunicación entre los sujetos y elaborar comportamientos. Sandoval (1997) enriquece estas funciones al agregar una cuarta: la valoración, que permite evaluar o enjuiciar hechos.

Abric (2001), en tanto, en su libro Prácticas sociales y representaciones, complementa el enfoque de Moscovici (1979) en torno a las funciones con dos elementos nuevos. El primero corresponde a la función justificativa, la cual ayuda a conformar justificaciones de acciones, de posturas y de comportamientos. 
El segundo refiere a la función identitaria, ya que las representaciones sociales facilitan el desarrollo de una identidad personal y social congruente con el sistema de normas sociales (Mugny \& Carugati, 1985, en Abric, 2001).

\section{Las múltiples dimensiones de la identidad}

La identidad es definida por Stuart Hall (2003) y por Erving Goffman $(1963,1981)$ como un elemento construido a través del tiempo, por lo que no es estática ni innata a los sujetos, sino que depende de las relaciones y las negociaciones que en relación con ella se generen en la interacción. De este modo, la identidad posee múltiples dimensiones, aunque cada una de ellas está atravesada por el contexto histórico y por la cultura.

Un elemento en el que es posible enfocarse es la dimensión discursiva de la identidad. Hall (2003) comprende que los discursos construyen constantemente la identidad, en un proceso que nunca acaba. A su entender, la identidad se materializa a través del levantamiento de fronteras simbólicas que se trazan mediante discursos, prácticas y posicionamientos que delimitan elementos grupales diferenciadores internos (homogéneos) y externos (heterogéneos). De acuerdo con este enfoque, la identidad nace a partir de una contingencia; necesita de una materialidad y de recursos simbólicos para sostenerse.

Por su parte, Goffman (1981) define la identidad como un elemento construido y negociado en las interacciones sociales. El autor aborda la identidad desde dos perspectivas. La primera refiere a una puesta en escena en donde las personas asumen roles dependiendo del escenario en el cual se desenvuelven; en este espacio, se busca generar una impresión positiva a través de signos verbales o de acciones que construyen una identidad positiva (Goffman, 1981). La segunda se relaciona con identidades estigmatizadas, las cuales nacen a partir de una noción compartida acerca de que quienes pertenecen a alguna categoría social deben situarse en un espacio determinado de acción, con lo cual las personas de dicha categoría se encuentran constreñidas, conflictuadas y/o en negociación constante con esta imposición (Goffman, 1963).

Respecto de la identidad personal, tanto Hall (2003) como Goffman (1963, 1981) concuerdan que esta se construye en relación con la posición que las personas 
tengan en el entramado social. Desde esta perspectiva, la identidad se encuentra estrechamente relacionada con los grupos de pertenencia en los que se desenvuelvan las personas, lo que a su vez influye en sus formas de interpretar el mundo y en sus conductas (Ovejero Bernal, 2000).

Conforme a lo expuesto, entre los conceptos de representación social y de identidad se observan similitudes, en tanto se construyen a partir de la interacción (física y/o verbal) de las personas, en un espacio determinado y en relación con objetos. Esta semejanza resulta relevante, ya que existen configuraciones simbólicas vinculadas a categorías que ocupan las personas, los roles, los lugares y los objetos desde antes de la entrada en escena de los sujetos, lo que se traduce en que al desempeñar un rol, habitar un lugar o utilizar un objeto, se adquieran estas configuraciones simbólicas, para reproducirlas y/o modificarlas. Asimismo, los símbolos adquiridos al relacionarse con estos objetos o lugares interfieren en la visión de mundo que los sujetos posean, en las formas en que son observados, en sus conductas y sus sentidos.

\section{Droga y consumo de marihuana}

Para la Organización Mundial de la Salud (OMS en SENDA, 2019b), droga es cualquier sustancia que al ser introducida al organismo afecte al sistema nervioso central, modificando la actividad emocional, psíquica y del organismo. Dentro de esta definición se encuentra la marihuana, que consiste en hojas secas y/o flores de la planta Cannabis Sativa. Considerada debido a ello una droga natural, contiene un número de compuestos cercano a 400, de los cuales el más conocido es el Tetra-Hidro-Cannabinol (THC) que produce cambios conductuales. En la actualidad, es común que exista marihuana prensada, a la cual se le agregan aditivos, solventes y otras sustancias que pueden provocar daños secundarios (SENDA, 2019b).

\section{Metodología}

Desde un enfoque cualitativo, el estudio busca comprender el fenómeno en su propio escenario y desde las significaciones que poseen quienes lo vivencian (Flick, 2007; Mayan, 2001). En función de este requerimiento, se buscó conocer las perspectivas 
que poseen los/as jóvenes sobre el consumo de marihuana en Erasmo Escala. Como se indicó, este espacio fue utilizado como referencia debido a que es un lugar de encuentro donde los/as estudiantes de la UAH se reúnen durante el día y parte de la noche, siendo habitual el consumo de alimentos, tabaco, alcohol y marihuana. ${ }^{1}$

Analizar las representaciones sociales de los/as jóvenes respecto al consumo de marihuana supuso un gran desafío, ya que se necesitó flexibilidad en el levantamiento de los datos y la construcción de un vínculo de confianza a fin de que los/as estudiantes pudiesen expresar los sentimientos, las sensaciones y las creencias a partir de los cuales comprenden, reproducen y reconstruyen el mundo, y que afectan sus actitudes y sus acciones ante el consumo de marihuana.

Conforme a la necesidad de flexibilidad, para el levantamiento de datos se optó por la técnica de entrevistas semiestructuradas, las cuales fueron realizadas entre el 7 de octubre y el 29 de noviembre de 2019. Este tipo de entrevista facilita que el actor se exprese de forma más espontánea, ya que el/la entrevistador/a no restringe lo que el/la entrevistado/a expresará sino que le otorga libertad en su comunicación. Por consiguiente, realizar entrevistas semiestructuradas permitió comprender emociones y conductas de los/as participantes tal como ellos/as las expresan a partir de sus experiencias y de su lenguaje cotidiano (Flick, 2007; Mayan, 2001).

Para conformar la muestra, se realizó un muestreo por cuotas, orientado a obtener un grupo de entrevistados/as con características preestablecidas acorde a las necesidades de la investigación (Flick, 2007). De esta manera, se buscó una heterogeneidad entre tres características: 1) carrera de estudio: sociología o antropología, 2) género, y 3) si consume o no consume marihuana. La conformación de la muestra no representativa de ocho estudiantes de pregrado, se resume en la siguiente tabla:

\begin{tabular}{|c|l|l|l|}
\hline \multicolumn{2}{|c|}{ SOCIOLOGÍA } & \multicolumn{2}{c|}{ ANTROPOLOGÍA } \\
\hline \multirow{2}{*}{ Masculino } & 1 Consumidor & \multirow{2}{*}{ Masculino } & 1 Consumidor \\
\cline { 2 - 2 } & 1 No consumidor & & 1 No consumidor \\
\hline \multirow{2}{*}{ Femenino } & 1 Consumidora & \multirow{2}{*}{ Femenino } & 1 Consumidora \\
\cline { 2 - 2 } & 1 No consumidora & & 1 No consumidora \\
\hline Total & $\mathbf{4}$ & & $\mathbf{4}$ \\
\hline
\end{tabular}

Tabla 2 | Distribución de la muestra por sexo, carrera y consumo Fuente: elaboración propia según muestreo 
La elección de las carreras de Sociología y de Antropología se debe a cuatro motivos. En primer lugar, existen formas diferenciadas por parte de los/as jóvenes de relacionarse con las drogas según el espacio en el que se ubiquen socialmente y la carrera estudiada (Morales y otros, 2011). Por esta razón, focalizarnos sobre dos carreras nos permite abarcar de forma más completa el entramado de significaciones de los/as estudiantes, en comparación a contemplar muchas carreras pero con menor cantidad de entrevistados/as por cada una.

En segundo lugar, es importante considerar que las fechas en las que se levantaron los datos corresponden a un período de intensa movilización y protesta social en Chile. Esto se tradujo en una menor disponibilidad de estudiantes para entrevistar, ya que muchos/as se vieron imposibilitados/as de llegar a la Universidad o debido a que participaron activamente del movimiento no podían centrarse en la entrevista, entre otros motivos.

En tercer lugar, al momento de realizar entrevistas en terreno, quienes estuvieron dispuestos a conversar abiertamente sobre el consumo de marihuana fueron estudiantes de las carreras descritas en el muestreo. Al respecto, cabe aclarar que la UAH posee escasa oferta de disciplinas consideradas como "Ciencias duras», ya que es una escuela orientada hacia el ámbito social (específicamente, se dictan 29 carreras «blandas» sobre 4 «duras»).

La técnica de análisis corresponde al análisis de contenido, reconocida por la posibilidad de aplicarse a diversos registros, por la sistematicidad en el trabajo de los datos y por su tratamiento riguroso (Bardin, 1996). Además, permite dilucidar y analizar el significado de los enunciados (Mayan, 2001), al contemplar tanto las palabras propiamente dichas por los/as entrevistados/as, como el contexto en el cual y respecto al cual estas palabras emergen (Abela, 2008). Considerar el contexto al analizar representaciones sociales es importante debido a que estas emergen desde la interacción, en relación con objetos y en espacios determinados.

Se informó a los/as entrevistados/as sobre los objetivos de la investigación y se aclaró que su participación era voluntaria. Además, se solicitó autorización para registrar la información mediante grabadora de voz y notas de campo. Finalmente, y tomando en cuenta el tema de investigación, se les ofreció la posibilidad de escoger un nombre ficticio (que diera cuenta de su género) con el cual se presentan sus palabras en la entrega de resultados. 


\section{Análisis y presentación de resultados}

A partir de los datos obtenidos, y en función del objetivo de investigación, se identificaron cuatro dimensiones de análisis que se resumen en la siguiente tabla:

\begin{tabular}{|c|c|}
\hline DIMENSIÓN & INDICADORES \\
\hline $\begin{array}{l}\text { 1| La universidad: } \\
\text { encuentro social } \\
\text { y uso de marihuana }\end{array}$ & $\begin{array}{l}\text { a. Naturalización del consumo. } \\
\text { b. Ampliación de las redes sociales e integración grupal. } \\
\text { c. Ausencia de control y facilidad de acceso. }\end{array}$ \\
\hline 2| Identidad & $\begin{array}{l}\text { a. Estigmas y estatus. } \\
\text { b. Carrera de procedencia: las ciencias sociales. } \\
\text { c. Identidad institucional y marihuana. }\end{array}$ \\
\hline $\begin{array}{l}\text { 3| Justificación } \\
\text { del consumo }\end{array}$ & $\begin{array}{l}\text { a. Inocuidad de la marihuana: vínculo con lo natural } \\
\text { y barrera con drogas duras/sintéticas. } \\
\text { b. Libertades y derecho. } \\
\text { c. La marihuana como «llave» a nuevas percepciones: } \\
\text { relajo y diversión. }\end{array}$ \\
\hline $\begin{array}{l}\text { 4| Resignificación } \\
\text { espacial }\end{array}$ & $\begin{array}{l}\text { a. Percepción del uso del espacio público. } \\
\text { b. Erasmo Escala como resistencia. }\end{array}$ \\
\hline
\end{tabular}

Tabla 3 | Dimensiones de análisis

Fuente: elaboración propia a partir de datos de campo

1| La universidad: encuentro social y uso de marihuana

\section{1.a Naturalización del consumo}

Los/as estudiantes reconocen que es difícil encontrarse con personas que no fumen marihuana en la Universidad, por lo que comprenden que el consumo se encuentra generalizado y que existe un uso homogéneo entre los/as estudiantes, de lo que se desprende que la disciplina que se estudie no es considerada un elemento significativo en las probabilidades de que un/a estudiante consuma marihuana. Al respecto, Isabel, estudiante de sociología, opina:

[...] ahora hay gente que es de derecho, de ingeniería comercial, ingenieros que también están consumiendo. Insisto, hay una mayor apertura que está abarcando más personas (Comunicación personal, estudiante de sociología del género femenino, 2019). 
En concordancia con otras investigaciones (Henao, 2011), los/as entrevistados/as comprenden que el consumo de marihuana se encuentra naturalizado por los/as estudiantes. Sobre esta naturalización, Tamara, estudiante de antropología, afirma:

Creo que es más probable un consumo sano si tu ambiente lo acepta, que si no lo acepta. [...] Creo que mientras más naturalizado está, también se puede hablar más y reflexionar más sobre el tema (Comunicación personal, estudiante de antropología del género femenino, 2019).

En su testimonio, Tamara reconoce que tanto la aceptación como la naturalización se valoran positivamente, ya que posibilitan hablar y reflexionar en torno a las consecuencias negativas y positivas del uso de marihuana.

\section{1.b Ampliación de las redes sociales e integración grupal}

El ingreso a la universidad genera expectativas y una disposición a conocer personas, tal como se evidencia en el testimonio de Juan:

[...] me imagino que uno no solamente viene a estudiar. Yo creo que uno también viene con la idea de conocer a más personas, y la mayoría de las personas fuman marihuana o han fumado marihuana (Comunicación personal, estudiante de antropología del género masculino, 2019).

Esta expectativa se cumple, ya que se amplían las relaciones sociales que los/as estudiantes poseen en comparación con etapas previas, y se reconoce que la universidad es un espacio de encuentro de muchas personas que en otras situaciones sería poco probable que se generara.

En este escenario, el uso de marihuana se entiende como un rito de amistad que alza una barrera simbólica entre quienes consumen y quienes no. En consecuencia, su consumo facilita las relaciones sociales y la integración grupal entre estudiantes consumidores, lo que reafirma los resultados de Henao (2011). En este contexto, se entiende que quienes fuman forman amistades fácilmente y generan lazos afectivos más duraderos.

Respecto a la construcción de lazos, llama la atención el testimonio de Juan, quien a pesar de no consumir reconoce el potencial de integración de la marihuana: 
Uno se da cuenta que los que fumaban, los que tenían consumo regular, eran los que se juntaban más, los que construían lazos afectivos mucho más profundos entre sí. En ese sentido, yo creo que la persona que no fuma se enfrentaría a ese dilema de si integrarse o no (Comunicación personal, estudiante de antropología del género masculino, 2019).

A pesar de que quienes fuman consideran que el uso de marihuana no genera una diferencia en la integración de las personas, quienes no consumen sí perciben esta barrera. Por lo tanto, la representación sobre el consumo conlleva a pensar que su uso permitirá encajar o sentirse parte de un grupo. El no participar de este rito produce incomodidad, un dilema personal de si integrarse o no hacerlo, y/o un cuestionamiento sobre si realmente se forma parte del grupo. Esto se manifiesta en el testimonio de Joaquín:

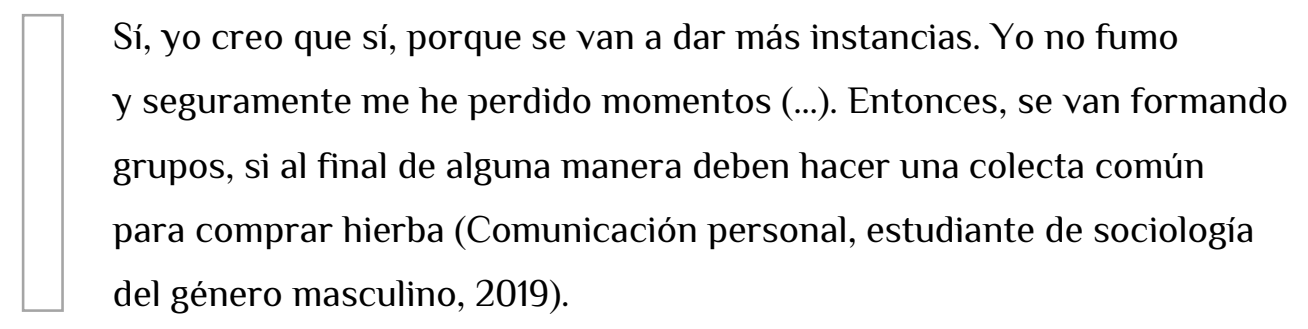

El testimonio reafirma que quienes no consumen marihuana perciben redes entre los/as fumadores/as, lo que produce la sensación de no compartir un vínculo fuerte o de que se generan distancias en relación con otros/as compañeros/as, al perderse de compartir instancias ocasionadas para juntarse a consumir. Esta percepción se evidencia en el siguiente testimonio de Pat:

[...] lo que hablaban harto era de marihuana. Hablaban solo de marihuana, pero los cabros que no consumían conocían razas, cosas que yo no sé. Y era como: «Ya, pero ¿tu fumas?», «No», «Ah, ya». No consumían, pero conocían sobre el tema para poder conversar (Comunicación personal, estudiante de antropología del género femenino, 2019).

Conocer sobre marihuana, a pesar de no consumirla, también permite integrarse. Debido a que es un tema en común que se manifiesta en las conversaciones, tener conocimientos sobre la marihuana facilita que los/as estudiantes puedan socializar. 
A pesar de ello, se compartiría un conocimiento común mas no la experiencia de un vínculo generado al compartir el rito de consumir marihuana.

\section{1.c Ausencia de control y facilidad de acceso}

De acuerdo con las entrevistas, la Universidad es percibida como un espacio en donde existen bajos niveles de control sobre los/as estudiantes, lo que torna posible consumir marihuana libremente en sus alrededores sin represalias institucionales. En este escenario, los/as jóvenes consideran que no es dificultoso adquirirla, aunque algunos/as son críticos/as respecto a la compra-venta, ya que consideran que esto conlleva a una criminalización del consumo dado que alimentan el tráfico de drogas. Al respecto, Ramón comenta:

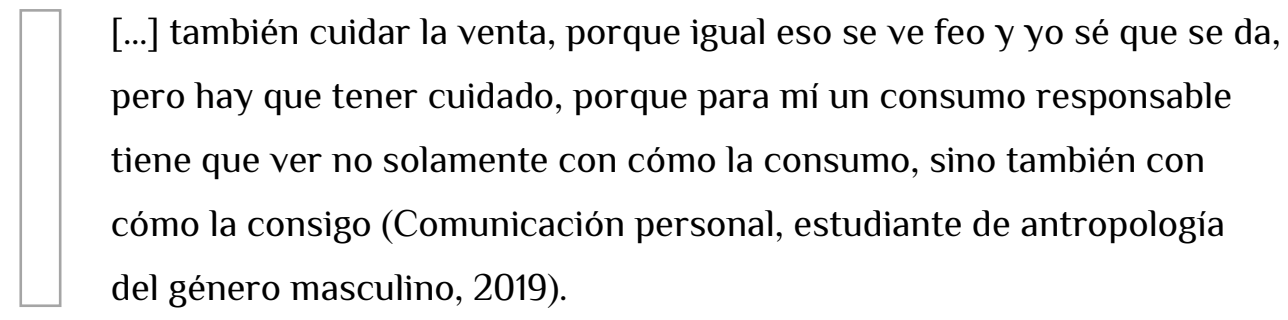

\section{2| Identidad}

\section{2.a Estigmas y estatus}

Los/as estudiantes comprenden que la edad es un factor que determina la percepción respecto a los/as consumidores/as. Así, es más probable que las personas mayores sean más críticas y estigmaticen más a los/as consumidores/as de marihuana que los/as jóvenes, quienes incluso pueden reconocer un estatus asociado al consumo. De esta manera, se enriquece la evidencia respecto a la estigmatización social que recae sobre los/as consumidores/as (Bulla y otros, 2010).

En este escenario, los/as jóvenes afirman que el Estado, a través de las campañas antidrogas, modeló en las generaciones pasadas estigmas sobre el/la consumidor/a de marihuana. Como se revela en el testimonio de Pat, estas campañas son percibidas como punitivas y no como preventivas: 
[...] en el fondo, el Estado nunca hizo buen trabajo de prevención, solo de castigo; de un castigo social. Además de que esté penalizado por ley, onda: «Tu fumas marihuana: eres tonto» (Comunicación personal, estudiante de antropología del género femenino, 2019).

Debido a estas percepciones acerca de los estigmas y los estatus de los/as consumidores/as, los/as estudiantes comprenden que el lugar donde las personas se desenvuelvan cotidianamente afecta el modo en que es percibido quien consume. Como se manifiesta en el siguiente testimonio de Bruno:

Yo creo que el que fuma marihuana comienza a fumar también porque su entorno está fumando y sabe dónde fumar o hablar de eso. Entonces, el estigma recibido es poco y quizás no se da donde la persona fuma, se da en otros espacios (Comunicación personal, estudiante de sociología del género masculino, 2019).

En consecuencia, como propone Goffman (1981), quien consume se «mueve estratégicamente» en concordancia al marco apreciativo de las personas; aprehende el lugar y el momento para consumir, y para definirse como consumidor/a. Los estigmas y el estatus asociados al uso de marihuana se resumen en la siguiente tabla:

\begin{tabular}{|l|l|}
\hline \multicolumn{1}{|c|}{ Estigmas } & \multicolumn{1}{c|}{ Estatus } \\
\hline Personas olvidadizas. & $\begin{array}{l}\text { Personas «cool» } \\
\text { (pueden controlar efectos y realizar su vida). }\end{array}$ \\
\hline Personas lentas. & $\begin{array}{l}\text { Personas «vacilonas» } \\
\text { (buen ánimo, disfrutan de encuentros de fiesta). }\end{array}$ \\
\hline Personas tontas. & $\begin{array}{l}\text { Personas «carretera» } \\
\text { (les gusta estar de fiesta). }\end{array}$ \\
\hline $\begin{array}{l}\text { Personas «flojas» } \\
\text { (se rehúsan a realizar tareas } \\
\text { o quehaceres). }\end{array}$ & $\begin{array}{l}\text { Personas «relajadas» } \\
\text { (no se complican con problemas). }\end{array}$ \\
\hline Personas con daños cognitivos. & Personas felices. \\
\hline Personas estúpidas. & Personas más espirituales. \\
\hline & Personas que saben divertirse. \\
\cline { 2 - 2 } & Personas que comparten. \\
\hline
\end{tabular}

Tabla 4 | Estigmas y estatus de personas consumidoras de marihuana Fuente: elaboración propia según categorización de estudiantes 


\section{2.b Carreras de procedencia: las ciencias sociales}

Estudiar sociología o antropología es reconocido por los/as estudiantes como un vinculante a estigmas relacionados con el uso de marihuana. Los/as jóvenes confiesan que en ocasiones han sido encasillados como consumidores/as de marihuana por ser estudiantes de ciencias sociales. Sobre estos prejuicios, Akemi, opina:

Es la visión prototípica que se tiene; prejuiciosa. Yo soy «la volada» de mi familia y todos mis primos fuman, jtodos! Pero yo soy a la que andan hueviando, onda: «Ah, andas con pitos en la mochila», porque tengo tatuajes y estudio sociología (Comunicación personal, estudiante de sociología del género femenino, 2019).

En este testimonio, se manifiesta el malestar de Akemi por ser estigmatizada, debido a la asimilación de su disciplina de estudio con el consumo de marihuana, a diferencia de lo que sucede con sus familiares, quienes también consumen.

\section{2.c Identidad institucional y marihuana}

Se reconoce que la construcción identitaria no es exclusiva de las personas, sino también de las instituciones. En este sentido, los/as estudiantes entienden que la UAH ha construido e intenta mantener una identidad relacionada con la excelencia, la inclusividad, el compromiso con la sociedad y la transformación social. Por lo tanto, el hecho de que se declarara «libre de humo» en 2017 es una forma de reafirmar esta identidad y de desligarse de los estigmas asociados a la marihuana. Esta percepción se manifiesta en el siguiente testimonio de Tamara:

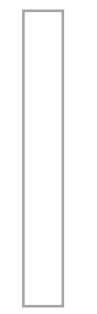

(...) en el fondo, si acá no se permite es porque se está cuidando la imagen hacia adentro y hacia afuera respecto del consumo de drogas al interior de la casa de estudios y, obviamente, hay una imagen negativa del consumo (Comunicación personal, estudiante de antropología del género femenino, 2019).

Asimismo, los/as entrevistados comprenden que el consumo de marihuana y de otras drogas, principalmente alcohol y tabaco, en Erasmo Escala afecta la credibilidad y la imagen institucional, lo que puede repercutir en el profesionalismo 
actual y futuro con que son asociados/as. En consecuencia, concuerdan que los/as estudiantes deben ayudar a proyectar una buena imagen institucional. Así lo refleja Joaquín:

Da vergüenza si tienes que invitar a alguien importante o hacer una entrevista con alguien; que vea que tu universidad es puro hueveo (...), cuando hay que dar una cara seria de la universidad (Comunicación personal, estudiante de sociología del género masculino, 2019).

\section{3| Justificación del consumo}

\section{3.a Inocuidad de la marihuana:}

\section{vínculo con lo natural y barrera con drogas duras/sintéticas}

La marihuana, habitualmente, es percibida como inofensiva; no afecta la salud y su consumo no es grave debido a que sus efectos son temporales. Sobre esta apreciación se sustenta gran parte de las justificaciones respecto a su uso, tanto en consumidores/as como en no consumidores/as. Al respecto, Joaquín, quien no consume marihuana, comenta:

Yo creo que cuando eres mayor de 21 no es nociva, porque al menos de lo que yo sé, no es un químico tóxico para el cuerpo, no genera grandes dificultades cognoscitivas después que se te pasa el efecto. La cocaína, por el contrario, es una droga que te vuelve violento, que te daña el cuerpo, te daña los pulmones (...), la cocaína es procesada. La marihuana, por el contrario, tiene químicos naturales (Comunicación personal, estudiante de sociología del género masculino, 2019).

Este discurso de inocuidad se produce a partir de su proveniencia, la naturaleza, debido a lo cual se genera un lazo entre lo natural y la sensación de que resulta inofensiva. Esta apreciación va cambiando, como observamos en el testimonio de Joaquín, a medida que la marihuana se mezcle con elementos sintéticos o se hable de drogas sintéticas: «el porro» o «paragua» (marihuana prensada) se concibe como dañino, el «nevado» (una mezcla de cocaína con marihuana) y el «mono» (una mezcla de pasta base con marihuana) son percibidos como altamente nocivos 
debido a que contienen «drogas duras», lo que se traduce en un rechazo. Sobre los efectos de estas drogas, Tamara comenta:

Hay cabros que fumaban porro siempre (...) y, obviamente, los efectos de ese consumo van a ser malos; en cambio, si tú fumas verde no.

(...) Obvio que si fumas con pasta es que estás consumiendo otra cosa; ya no estamos hablando de marihuana, estamos hablando de una droga, o sea la pasta base es muy destructiva y es muy adictiva (Comunicación personal, estudiante de antropología del género femenino, 2019).

Testimonios como el de Tamara demuestran que mientras más efectos negativos son percibidos, mayor es el rechazo a la sustancia. Además, existe una relación entre droga dura y menos control de sus efectos, mayores niveles de violencia, estigma social y dependencia.

\section{3.b Libertades y derecho}

La palabra libertad convoca tanto a quienes se encuentran de acuerdo con el consumo de marihuana como a quienes se encuentran en desacuerdo. Quienes lo aceptan, entienden que consumir corresponde a una decisión personal que debe tomarse de manera responsable; por lo tanto, poder consumir marihuana corresponde a un derecho a partir de las libertades de las personas. Esta percepción se observa, también, en sujetos que no usan marihuana. Como señala Joaquín:

Creo que está bien que uno tenga derecho a consumir donde quiera y cuando quiera (...). Puedes fumar un pito en la Universidad y da lo mismo, porque es tu decisión (...). Vas a andar volado y no vas a hueviar a nadie (Comunicación personal, estudiante de sociología del género masculino, 2019).

En la aceptación del consumo existen matices. Las posturas van desde una total apertura a otras que sostienen algunas limitaciones, entre ellas, tener una edad mínima de 21 años, debido a que previamente afecta el desarrollo neurológico. En torno al límite de edad, Ramón expone lo siguiente: 
En términos neuronales o neurológicos, no sé cómo se dice, pero creo que tiene efectos, sobre todo, si se consume antes de tener el pleno desarrollo del cuerpo, que está por ahí entre los 21 años de edad (Comunicación personal, estudiante de antropología del género masculino, 2019).

Otra restricción se vincula con tener información respecto a los posibles problemas que genera el consumo de marihuana, ya que existe la sensación de que la marihuana es un tema tabú debido a que se encuentra penalizada por ley, lo que impide su tematización. Debido a esto, al momento de comenzar a utilizar marihuana, las personas habitualmente no poseen conocimientos acerca de los riesgos que enfrentan. Al respecto, Akemi comenta:

Debería haber una forma de poder advertir sobre los riesgos que sí puede llevar (...). Como que nadie te los dice, y si te los dicen es como: «Es pésimo, no lo hagas». No es: «Hazlo bajo tu responsabilidad y ten esto en cuenta». (Comunicación personal, estudiante de sociología del género femenino, 2019).

En tanto, quienes rechazan el consumo de marihuana también justifican su posición a partir de las libertades individuales, especialmente, cuando se consume en espacios públicos, como ocurre en Erasmo Escala. En estos casos, el problema se debe al olor, que ingresa a las dependencias de la Universidad e impide un desarrollo normal de actividades. Esta molestia es manifestada, contundentemente, por personas no consumidoras, tal como se observa en el siguiente testimonio de Isabel:

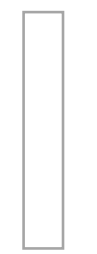

[...] es una falta de respeto que otras personas que no consumimos tengamos que tragarnos ese olor. Cuando uno está en clases entran los olores, incluso dentro de la sala, lo cual es una falta de respeto (Comunicación personal, estudiante de sociología del género femenino, 2019).

En este sentido, también se perciben problemas derivados del consumo que afectan en forma individual a quien consume. Una problemática corresponde a la dependencia y se asocia con una pérdida de la capacidad para divertirse, para distraerse y/o para relajarse estando lúcido.

Este riesgo y, por tanto, la justificación del rechazo del consumo de marihuana, se manifiesta en la siguiente declaración de Isabel: 
La gente llega a necesitar este tipo de consumo para poder reírse, para poder dispersarse, y no tiene la capacidad de no sé... De sentarse sin estar con alcohol, sin marihuana, sin nada, con un vaso de agua, incluso. Simplemente, conversar y reírse (Comunicación personal, estudiante de sociología del género femenino, 2019).

Finalmente, los/as jóvenes rechazan el uso de otras drogas al momento de estar bajo los efectos de la marihuana debido a que consideran que el poli consumo de drogas -específicamente, la mezcla de alcohol y de marihuana- genera situaciones de violencia (Rodríguez y otros, 2006). En consecuencia, se percibe el espacio de Erasmo Escala no solo como un ámbito para socializar, sino también como un lugar donde pueden ocurrir hechos violentos. Respecto a estas situaciones, Bruno comenta:

Hubo un momento en que Erasmo fue muy problemático, hubo mucha violencia (...), hubo gente agarrándose a cachos, botellazos y llegaron los pacos. Cuando uno toma mucho copete, sale mucha de la rabia que tiene uno (...), y así se llega a los botellazos (Comunicación personal, estudiante de sociología del género masculino, 2019).

\section{3.c La marihuana como «llave» a nuevas percepciones: el relajo y la diversión}

Para algunos/as entrevistados/as, la marihuana es como una «llave» que permite entrar a estados perceptivos que no se tienen cuando se está lúcido; por ejemplo, potenciar la introspección al alcanzar otro grado de conciencia. Además, entienden que en ocasiones permite reflexionar mejor u observar las cosas desde otra perspectiva, lo que facilita llegar a nuevas conclusiones y ayuda a estudiar, a concentrarse e, incluso, como indica Juan, a experimentar relaciones sexuales más placenteras:

Yo creo que a la gente le puede servir para tener otra percepción y escribir, dibujar, expresarse artísticamente, estudiar y, bueno, también disfrutar de un carrete o también del sexo (Comunicación personal, estudiante de antropología del género masculino, 2019).

Debido a esta «apertura perceptiva», los/as estudiantes entienden que el uso de marihuana facilita alcanzar estados de relajación, de distracción y/o de diversión; 
en concordancia con otras investigaciones realizadas (García y otros, 2010). Esta facilidad para relajarse y/o para divertirse ayuda a alivianar el estrés y la carga emocional que se genera a partir de la sensación de altos niveles de carga académica, entendiendo, incluso, que el consumo de marihuana permite adaptarse al ritmo universitario. Esta asociación del consumo con sensaciones de relajo, de diversión y con la posibilidad de adaptarse a las demandas universitarias se evidencia en el siguiente testimonio de Bruno:

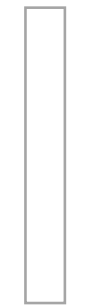

La marihuana hace un resorte para que el estudiante pueda divertirse, relajarse y, después, tener la tranquilidad para ver qué hacer con tanta carga académica y, en el fondo, adaptarse al ritmo de vida que tiene el chileno promedio hoy en día (Comunicación personal, estudiante de sociología del género masculino, 2019).

A pesar de que los/as jóvenes reconocen que el consumo de marihuana aliviana el estrés académico, son críticos respecto a esta justificación, debido a que estos problemas se generan por cargas académicas que consideran excesivas. En este escenario, si bien se utiliza y se acepta el uso de marihuana y de medicamentos para superar estas emociones, los/as entrevistados/as concuerdan que con ello no se resuelve el problema real y se pasa por alto el problema de salud que se genera en los/as estudiantes. Esto se evidencia en el testimonio aportado por Ramón:

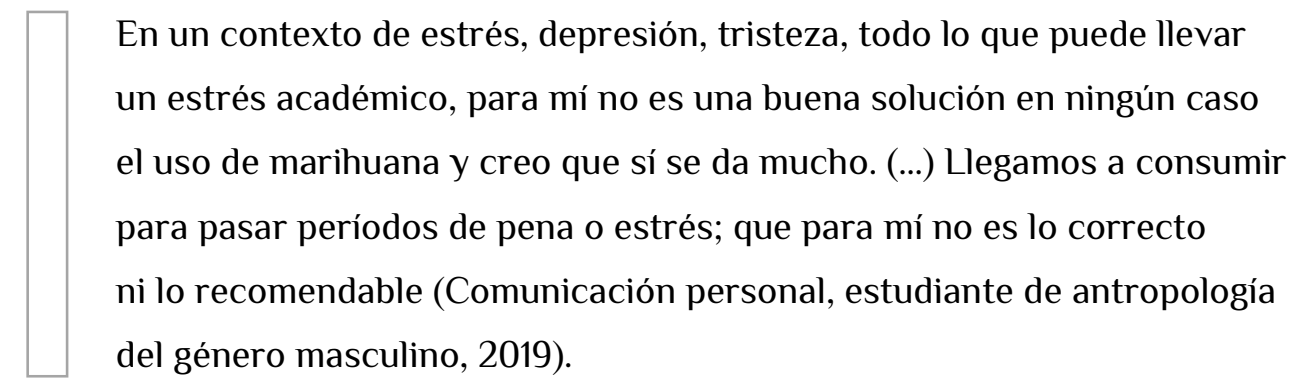

\section{4| Resignificación espacial}

\section{4.a Percepción del uso del espacio público}

Los/as jóvenes reconocen que existe una percepción compartida en el sentido común de la sociedad de que en el espacio público debe existir tranquilidad, tránsito libre, tanto para vehículos como para personas. Por lo tanto, el consumo de marihuana, alcohol y tabaco en Erasmo Escala tensiona estas nociones de 
tranquilidad, y se reconoce que la forma de compartir no es adecuada al lugar. Esta percepción se refleja en el testimonio de Isabel respecto al uso de Erasmo Escala:

Sí, se debe evitar el consumo y el supuesto compartir. O sea, no es supuesto, sí comparten, pero de una forma que no es la correcta para el espacio en que se está (Comunicación personal, estudiante de sociología del género femenino, 2019).

\section{4.b Erasmo Escala como resistencia}

Los/as jóvenes entienden que se ha utilizado un espacio que no es apto para compartir, lo que puede generar molestas en estudiantes, vecinos/as, profesores/as y/o funcionarios/as. A pesar de ello, comprenden que su uso es en respuesta a una necesidad no satisfecha por la Universidad, la posibilidad de contar con un espacio seguro y regulado para compartir, y son propositivos sobre las formas de transformar este espacio para la comunidad:

Deberían urbanizar Erasmo, (...) para que, por último, si quieres fumar marihuana puedas fumar sentado y no tirado en el piso, o si quieres reunirte con tus amigos a conversar de lo que sea, que al menos sea un espacio tranquilo, regulado, que no lleguen los pacos y que no se pongan a escuchar música fuerte (Comunicación personal, estudiante de sociología del género masculino, 2019).

Debido a que no existe un espacio regulado para compartir, algunos/as estudiantes valoran que los/as jóvenes utilicen un lugar que no fue creado para ello y transformen su uso. No obstante, concuerdan que debe ser utilizado de forma responsable y consciente, principalmente en temas de higiene, ya que su utilización no debiese generar más trabajo para otras personas. Esta valoración es compartida por Pat, quien entiende a Erasmo Escala como un espacio de resistencia:

Con consideración, pero no consideración por el cemento o por el pedazo de muralla, sino porque si tu fumas cigarro y no lo pones en un basurero lo va a recoger una persona y lo va a botar. No tiene por qué venir una persona, que por lo general son personas que trabajan en la Universidad, no tiene por qué salir de la Universidad a recoger tu mierda (Comunicación personal, estudiante de antropología del género femenino, 2019). 
En consecuencia, la utilización de espacios para distraerse trae aparejadas preocupaciones respecto de su uso, en la medida en que no debiese generar perjuicios a terceros.

\section{Conclusiones}

En las representaciones sociales que tienen los/as estudiantes sobre el consumo de marihuana subyacen elementos simbólicos y objetivaciones, producidas socialmente, que afectan la manera en que los/as jóvenes perciben y se posicionan en torno al uso de la marihuana, y que construyen un marco normativo con relación a ella. Los/as jóvenes habitan estas redes simbólicas y construyen un lenguaje coherente al marco normativo en torno al uso de esta droga. Estas redes son complejas, lo que se traduce en que existan diferentes posicionamientos en relación con el uso de marihuana en los/as estudiantes y que este pueda modificarse con el tiempo.

Es importante destacar que la representación sobre el consumo de marihuana varía en función de si la persona consume o no. De este modo, es más probable que las personas que no consumen carguen con más representaciones negativas, visualicen mayor cantidad de peligros y/o riesgos más altos en relación con personas que sí usan esta droga. Pese a esto, el consumo se encuentra ampliamente aceptado.

En este contexto, el hecho de que al ingresar a la universidad los/as jóvenes tengan altas expectativas de generar nuevas redes sociales implica que puedan optar por fumar marihuana para integrarse a grupos. Además, debido a que en estos espacios su consumo se encuentra naturalizado, es de fácil acceso y no se castiga su uso ni social ni institucionalmente, se convierte en una posibilidad viable para socializar.

La naturalización y la aceptación del consumo se basan en una percepción baja sobre los riesgos que genera el uso de marihuana. Esta percepción se produce debido a la proveniencia natural de la droga y se defiende a partir de un contraste con drogas sintéticas o «duras», lo que implica que pocos/as estudiantes reconozcan o se informen acerca de los posibles riesgos que conlleva su consumo y que exista una sensación de desconocimiento sobre los peligros asociados. 
Los/as estudiantes reconocen la presencia de estigmas asociados a los/as consumidores/as, mas no consideran que se expresen entre compañeros/as sino con personas mayores. Entonces, la edad de las personas afecta el modo en que perciben a quienes consumen marihuana; mientras mayores son las personas, más son los estigmas sobre el uso de marihuana, los cuales disminuyen para las generaciones más jóvenes, pudiendo, incluso, transformarse en una característica identitaria positiva.

Los/as jóvenes, habitualmente, justifican su consumo a partir de la diversión o de alivianar el estrés. En el espacio universitario, el estrés que producen las exigencias académicas es considerado un factor que gatilla el deseo de fumar marihuana, por lo que atravesar períodos de estrés académico es compensado con momentos de diversión y de relajo. Esta justificación implica, como reconocen algunos/as jóvenes, que no se trabajen o no se enfrenten los problemas de fondo que se generan en el contexto universitario: depresión, situaciones de agobio y cargas académicas excesivas.

Los/as estudiantes reconocen que hace uso de un espacio público de una forma que no corresponde, pero justifican su actuar a partir de lo que entienden como una necesidad no resuelta por parte de la UAH. La prohibición de fumar dentro de la Universidad es entendida como una irresponsabilidad de la institución debido a que no soluciona sus necesidades: contar con un espacio seguro para compartir más allá de lo académico. Esto trajo consigo una resignificación del espacio público, como forma de resistencia ante la prohibición establecida por la institución, a partir de entender que en la medida en que no existan espacios de convivencia al interior de las dependencias institucionales se seguirán utilizando espacios inadecuados.

Regular el espacio es considerado importante, debido a que el consumo de marihuana $-y$, en ocasiones, de tabaco- se encuentra profundamente vinculado al relajo, la distracción, la integración grupal y la generación de amistad. Esto implica que está presente en la mayoría de las actividades extracadémicas mediante las cuales los/as jóvenes se divierten o se distraen. En concordancia con las representaciones relevadas, es viable crear zonas seguras para compartir, entendiendo que en la universidad los/as estudiantes no solo se desarrollan académicamente, así como contemplar zonas habilitadas para fumadores/as, considerando la congruencia con el cuidado, la higiene y la formalidad de la imagen 
que se debe exponer hacia el interior y hacia el exterior de la Universidad. Además, es pertinente crear, reforzar y/o difundir otras formas de relajo y de diversión extraacadémicas para el estudiantado.

Finalmente, esta investigación abre la puerta a nuevos estudios sobre representaciones sociales de jóvenes, estudiantes o no, acerca del consumo de drogas. Sabemos que la posición en la que se ubique la persona determina las significaciones en torno al fenómeno, por lo que considerar otras universidades u otras carreras de estudios puede brindar una perspectiva más amplia sobre las representaciones de las juventudes y aportar una base para que las instituciones, tanto académicas como gubernamentales, puedan acercarse y responsabilizarse de una forma congruente con el consumo de marihuana y de drogas, en general.

\section{Referencias}

Abela, J. A. (2008). Las técnicas de análisis de contenido. Una revisión actualizada. Granada, España: Universidad de Granada.

Abric, J.-C. (2001). Prácticas sociales y representaciones. Ciudad de México, México: Covoacan.

Aguilera Ruiz, O. (2009). Los estudios sobre juventud en Chile. Coordenadas para un Estado del Arte. Última Década, 17(31), 109-127. Recuperado de https://ultimadecada.uchile.cl/index.php/UD/article/view/56056

Araya, S. (2002). Las representaciones sociales: ejes teóricos para su discusión. San José, Costa Rica: Facultad Latinoamericana de Ciencias Sociales (FLACSO). Recuperado de https://www.academia.edu/24267404/Las_representaciones_sociales_Ejes_ te\%C3\%B3ricos_para_su_discusi\%C3\%B3n

Banchs, M. (1986). Concepto de representaciones sociales: análisis comparativo. Revista costarricense de psicología, (89). 27-40.

Bardin, L. (1996). Análisis de contenido. Madrid, España: Akal. 
Barrera-Herrera, A. y Vinet, E. (2017). Adultez emergente y características culturales de la etapa en universitarios chilenos. Terapia Psicológica, 35(1), 47-56. Recuperado de http://teps.cl/index.php/teps/article/view/152

Berger, P. y Luckmann, T. (1968). La construcción social de la realidad. Ciudad Autónoma de Buenos Aires, Argentina: Amorrortu.

Bourdieu, P., Passeron, J. C., Melendres, J. y Subirats, M. (1981). La reproducción. Elementos para una teoría del sistema de enseñanza. Barcelona, España: Laia.

Bulla, A., Vásquez, A., Güichá, A., Manrique-Abril, F. y Ospina, J. (2010). Representaciones sociales del consumo de marihuana en estudiantes universitarios. Psychologia. Avances de la disciplina, 4(1), 89-101. https://doi.org/10.21500/19002386.1161

Cáceres, D., Salazar, I., Varela, M. y Tovar, J. (2006). Consumo de drogas en jóvenes universitarios y su relación de riesgo y protección con los factores psicosociales. Univ. Psichol., 5(3), 521-534. Recuperado de https://revistas.javeriana.edu.co/index.php/revPsycho/article/view/458

Camarotti, A. y Kornblit, A. (2005). Representaciones sociales y prácticas de consumo del éxtasis. Convergencia, 12(38), 313-333. Recuperado de http://www.scielo.org.mx/scielo.php?script=sci_arttext\&pid=\$140514352005000200313\&lng=es\&tlng=es

Cazenave, A., Saavedra, W., Huerta, P., Mendoza, C. y Aguirre, C. (2017). Consumo de marihuana en jóvenes universitarios: percepción de los pares. Ciencia y Enfermería, 23(1), 15-24. http://dx.doi.org/10.4067/S071795532017000100015

Consejo Nacional para el Control de Estupefacientes (CONACE). (2008). Representaciones sociales sobre el consumo de drogas de la(s) juventud(es) chilena. Santiago de Chile, Chile. 
Faúndez, X. y Vinet, E. V. (2009). Tipología empírica de adolescentes consumidores de drogas según el MACl. Psykhe, 18(2), 19-35.

Recuperado de

https://scielo.conicyt.cl/scielo.php?script=sci_arttext\&pid=S0718-

22282009000200002

Flick, U. (2007). El diseño de la investigación cualitativa.

Madrid, España: Morata.

García, A., Torres, D., Barrios, F., Vanessa, P., Rubio, A. y Ugarte, L. (2010).

Representación social del consumo de marihuana en un grupo de jóvenes universitarios consumidores de la Universidad Tecnológica de Pereira.

Revista Médica de Risaralda, 16(2), 25-30. Recuperado de

https://revistas.utp.edu.co/index.php/revistamedica/article/view/811

Goffman, E. (1963). Estigma. La identidad deteriorada.

Ciudad Autónoma de Buenos Aires, Argentina: Amorrortu.

Goffman, E. (1981). La presentación de la persona en la vida cotidiana.

Ciudad Autónoma de Buenos Aires, Argentina: Amorrortu.

Hall, S. (2003). Introducción. ¿Quién necesita identidad? En S. Hall y P. du Gay, Cuestiones de identidad cultural.

Ciudad Autónoma de Buenos Aires, Argentina: Amorrortu.

Henao, S. (2011). Representaciones sociales del consumo de drogas en un contexto universitario, Medellín, Colombia, 2000.

Revista Fac. Nac. Salud Pública, 30(1), 26-37. Recuperado de http://aprendeenlinea.udea.edu.co/revistas/index.php/fnsp/article/view/ 10587

Ibáñez, T. (1988). Ideologías de la vida cotidiana.

Barcelona, España: Sendai. 
Jiménez-Muro Franco, A., Beamonte, A., Marqueta, A., Gargallo, P. y Nerin, I. (2009). Consumo de drogas en estudiantes universitarios de primer curso. Adicciones, 21(1), 21-28.

http://dx.doi.org/10.20882/adicciones.248

Jodelet. D. (1984). La representación social: fenómenos, conceptos y teoría. En S. Moscovici, Psicología social II. Pensamiento y vida social. Psicología social y problemas sociales. Ciudad Autónoma de Buenos Aires, Argentina: Paidós.

López Pinedo, M. (2012). Influencia del consumo de drogas en los estudiantes universitarios. Revista Estomatológica Herediana, 22(4), 247-256. https://doi.org/10.20453/reh.v22i4.97

Mayan, M. (2001). Una introducción a los métodos cualitativos. Módulo de entrenamiento para estudiantes y profesionales. Itzapalapa, México: International Institute for Qualitative Methodology.

Morales, G., Del Valle, C., Belmar, C., Orellana, Y., Soto, A. e Ivanovic, D. (2011). Prevalencia de consumo de drogas en estudiantes universitarios que cursan primer y cuarto año. Revista Médica de Chile, 139(12), 1573-1580. http://dx.doi.org/10.4067/S0034-98872011001200006

Moscovici, S. (1979). El psicoanálisis, su imagen y su público.

Ciudad Autónoma de Buenos Aires, Argentina: Huemul.

OEA y CICAD. (2019). Informe sobre el consumo de drogas en las Américas 2019. Washington D.C., Estados Unidos: Organización de los Estados Americanos.

Ovejero Bernal, A. (2000). La adicción como búsqueda de identidad. Una base teórica psicosocial para una intervención eficaz. Psychosocial Intervention, (9), 199-215. Recuperado de https://dialnet.unirioja.es/servlet/articulo?codigo=2008348 
Ramírez, J., Cunningham, J., Brands, B., Strike, C. y Miotto, M. (2009).

Normas percibidas por los estudiantes universitarios acerca de sus pares y uso de drogas en una universidad en Chile. Revista Latino-Americana de Enfermagem, 17(núm. especial), 907-912. http://dx.doi.org/10.1590/S0104-11692009000700023

Rodríguez, J., Fernández, A., Hernández, E., y Ramírez, S. (2006). Conductas agresivas, consumo de drogas e intentos de suicidio en jóvenes universitarios. Terapia Psicológica, 24(1), 63-69. Recuperado de https://www.redalyc.org/articulo.oa?id=78524107

Saldivia, C. y Vizcarra, B. (2012). Consumo de drogas y violencia en el noviazgo en estudiantes universitarios del sur de Chile. Terapia Psicológica, 30(2), 43-49. Recuperado de http://teps.cl/index.php/teps/article/view/122

Servicio Nacional para la Prevención y Rehabilitación del Consumo de Drogas y Alcohol (SENDA). (2017). Décimo Segundo Estudio Nacional de Drogas en Población General de Chile, 2017. Santiago de Chile, Chile: Ministerio del Interior y Seguridad Pública.

Servicio Nacional para la Prevención y Rehabilitación del Consumo de Drogas y Alcohol (SENDA). (2019a). Primer estudio de drogas en educación superior [Presentación]. Santiago de Chile, Chile: Ministerio del Interior y Seguridad Pública. Recuperado de http://www.senda.gob.cl/wpcontent/uploads/2019/05/2019_05_23_PPT_Ed_SuperiorEstudiosOK.pdf

Servicio Nacional para la Prevención y Rehabilitación del Consumo de Drogas y Alcohol (SENDA). (2019b). Información sobre drogas. Glosario de términos. Recuperado de https://www.senda.gob.cl/informacion-sobredrogas/conoce-mas-sobre-las-drogas/glosario/terminos-g-a-la-m/

Silva, J. (2002). Juventud y tribus urbanas: en búsqueda de la identidad. Última Década, 10(17), 117-130. http://dx.doi.org/10.4067/S071822362002000200006 
Vázquez, V., \& Castro, R. (2009). Masculinidad hegemónica, violencia y consumo de alcohol en el medio universitario. Revista Mexicana de Investigación Educativa, 14(42), 701-719. Recuperado de http://www.scielo.org.mx/scielo.php?script=sci_arttext\&pid=\$140566662009000300003

Nota

1 Este lugar nace como punto de encuentro debido a que en 2017 la Universidad fue declarada institución libre de humo. 